\title{
Protée
}

\section{Qui dit quoi?}

\section{Francesca Caruana}

Volume 26, numéro 3, 1998

\section{Logique de l'icône}

URI : https://id.erudit.org/iderudit/030521ar

DOI : https://doi.org/10.7202/030521ar

Aller au sommaire du numéro

\section{Éditeur(s)}

Département des arts et lettres - Université du Québec à Chicoutimi

\section{ISSN}

0300-3523 (imprimé)

1708-2307 (numérique)

Découvrir la revue

\section{Citer cet article}

Caruana, F. (1998). Qui dit quoi? Protée, 26(3), 7-16.

https://doi.org/10.7202/030521ar

\section{Résumé de l'article}

Dans le domainede l'imitation, l'art contemporain pourrait avoir l'apanage de l'imitation dans la mesure où la plupart du temps il n'offre pas de difficultés techniques majeures à être reproduit et pousserait à reconsidérer la problématique du faux. En réalité, la complexité des motivations esthétiques indexée au contexte socio-historique montre que les artistes, qui ont "imité " des manières, des styles, ont procédé à une relève critique de la question de l'imitation. C'est en soumettant principalement deux mouvements à l'analyse sémiotique, l'hyperréalisme et le surréalisme, que la proposition s'est formée d'aborder la question du faux sous l'angle du réel de la création.
Ce document est protégé par la loi sur le droit d'auteur. L'utilisation des services d'Érudit (y compris la reproduction) est assujettie à sa politique d'utilisation que vous pouvez consulter en ligne.

https://apropos.erudit.org/fr/usagers/politique-dutilisation/ 


\section{ounorroust}

FRANCESCA CARUANA

\section{SI C'EST VRAIMENT FAUX... ALORS!}

En peinture, et plus spécifiquement en arts plastiques, l'interrogation maintes fois posée par la problématique du faux implique la réciprocité logique d'un vrai. La question sur la réalité de l'un et de l'autre ayant été traitée sous bien des aspects, elle ne suscitera pas ici un développement supplémentaire, elle présentera plutôt une réflexion sur les composantes iconiques de l'imitation d'une part et, d'autre part, une réflexion sur les effets que ces composantes impliquent dans l'interprétation, lorsque le spectateur est en situation de reconnaître quelque chose. Car, au-delà de l'association implicite vrai/faux, le projet veut mettre en évidence, au sein même de la production picturale, du point de vue de l'interprétation, la fonction sémiotique relative aux éléments de ressemblance, spontanément intégrés par le regard du spectateur soumis, dans ces conditions, à l'obligation de différentiation. Mais ce jugement spontané produit par des éléments qualitatifs laisse supposer un état antérieur, originel, naturel, apparemment vrai, qui aurait le privilège de tenir lieu de référence. Il conviendra donc de définir cet état antérieur, dont les effets semblent fondés sur le seul argument de la sensibilité, selon que l'éthique qui la gouverne la dissocie ou non d'un état provisoire des connaissances.

L'histoire de l'esthétique propose des séries d'œuvres qui ont représenté la nature, les objets du monde, d'après les lois de l'imitation telles qu'elles étaient définies par les différents contextes socio-historiques (de l'art antique à l'art classique en passant par l'art moderne, les canons de l'imitation n'ont cessé d'être modifiés, et correspondaient à l'investissement mythique, religieux, voire économique que les sociétés respectives faisaient surgir). Les formes de l'apparence, via les objets qui les représentent, qui déterminent ultérieurement les ancrages interprétatifs de ce rapport implicite - c'est-à-dire l'objet et sa représentation mimétique -, se trouvant être à l'origine de valeurs moralisantes, à l'origine d'argumentation religieuse ou mythique, sont analysées à l'aide de la topologie de l'icône telle que Peirce l'a établie. Jusque-là, ces ancrages interprétatifs ont été mis en exergue à la faveur d'une critique hiérarchisante, qui en a catégorisé les effets: du plus vrai à l'absolument faux, à l'archi(archê)-faux, en passant par la 
copie, le clone, le double, la série, le modèle, le multiple, l'épreuve... De l'objet unique à l'objet plagié, quelle sorte d'altération affecte donc l'objet pour qu'on puisse dire qu'il est faux? À quelle sorte de réalité mimétique, répétitive, la ressemblance nous renvoie-t-elle? Toutes ces questions concernent une interprétation historico-esthétique du monde.

Mais qu'advient-il de l'interprétation picturale, lorsqu'elle est déjà investie de la problématique de l'imitation et que la peinture cherche à la dépasser ou à la réinterpréter comme dans le cas du surréalisme ou de l'hyperréalisme? C'est précisément ce que je me propose d'examiner.

Les sujets soumis à l'analyse relèveront des expressions picturales citées, sachant qu'elles présupposent acquis par le lecteur que tout jugement moralisant sur la valeur des œuvres en fonction de la réussite de l'imitation est écarté. Les qualités iconiques des diverses expressions sont observées de manière comparative afin de caractériser les sources de l'interprétation. En effet, l'hypothèse que je pose tentera de soutenir que des qualités «intrinsèques» de l'œuvre perçues ou ignorées par le spectateur dépend une vérité esthétique gouvernée par une loi d'interprétation. On peut annoncer d'ores et déjà que la loi esthétique sera du côté de celui qui, d'une certaine manière, aura saisi les qualités inhérentes à l'œuvre. Il faut toutefois maintenir une précaution méthodologique qui n'établira pas de lien de cause à effet entre le fait de percevoir et la vérité de la loi esthétique. L'objet considéré est celui de l'inscription plastique, soit un ensemble de signes qui ne sont pas encore des signes mais dont la nature est de représenter leur objet: les pictorhèmes ${ }^{1}$.

Pour «illustrer» cette relation entre les qualités de l'œuvre - ce qui semble gouverner une vérité esthétique et la loi d'interprétation -, je propose que nous passions du symbolique au réel, en jouant d'une éventualité quelque peu extrême mais qui, à mon sens, fait se représenter au mieux ce que l'être humain élabore quand, remontant vers l'origine, il cherche une vérité: je fais donc l'hypothèse d'une posture somme toute assez ordinaire, celle de l'aspiration à la vérité, représentée par la place la plus vide qui soit (sur le plan symbolique), celle de Dieu, théologiquement nommée comme vraie, puisque susceptible d'être remplie par toutes sortes de prédicats satisfaisant à une vision non avouée mais idéale du monde - toute représentation venant décliner au plus près cette forme de postulat. Tout se passe, telle l'image d'une pyramide symbolique:

- Il y aurait Dieu, ensuite celui qui lui est le plus ressemblant, le Saint, puis celui qui lui est le plus proche, l'homme. En supposant cette filiation, on obtient un point d'origine qui n'a d'autre intérêt que la prise en charge $d u$ vrai, c'est-à-dire d'un postulat idéal, même si l'on repère très vite qu'il ne peut être qu'imaginaire. Mais continuons à refaire le monde. En lieu et place de Dieu, l'homme pose comme garantie de Son œuvre parfaite la nature, entité inamovible de la pensée commune et dont les effets sont omniprésents. L'homme dispose dès lors d'un univers de références maitrisable et, à quiconque contesterait cet état de garantie, il deviendrait difficile de faire la preuve de l'inamovibilité de la nature. Mais au-delà, la preuve de quoi? C'est ici qu'un développement se justifie. L'artiste pour qui l'inclination à imiter ne répondra pas à la restitution d'une nature identique, comme si elle était vraie, pour qui l'inclination ne sera pas de se laisser prendre à un ordre d'origine, l'artiste donc ne se réduira pas à modéliser la nature, il rendra compte de quelque chose d'autre, de tout aussi vrai, mais la chose ellemême sera produite dans un domaine singulier. Il s'agira d'une chose réelle, vraie et non universelle. Ce glissement essentiel d'une valeur à une autre délimite l'écart entre les niveaux de l'interprétation.

L'interprétation, dont la préoccupation est la recherche $d u$ vrai, comble une relation iconique entre l'œuvre et son modèle pour ce qui est de la ressemblance, indiciaire pour le modèle auquel elle renvoie et symbolique pour l'impossibilité d'être l'objet en question. La deuxième trichotomie du signe définie par Peirce rend compte de ses trois aspects catégoriels: l'icône (catégorie de la priméité), l'indice (catégorie de la secondéité), le symbole (catégorie de la 
tiercéité) ${ }^{2}$. L'interprétation produite selon des critères argumentés (qui dépendent de la troisième

trichotomie, rhème, dicisigne et argument) ${ }^{3}$ révèle un jugement dont les éléments subordonnés à une articulation logique sont vérifiables en contexte. Dans ce cas d'espèce, la perception primitivement saisie chez le spectateur entraîne spontanément dès l'observation un jugement discriminant et il favorise ainsi, tout aussi spontanément, ce qui a été reconnu comme juste, de manière quasiment paradoxale. Il reste donc à envisager un autre type de rapport, entre le vrai et le juste, si le faux se pose comme un point limite de la représentation. Le rapport dont il s'agit est un rapport métaphorique. Autant d'icônes à explorer pour en extraire les états et les effets sémiotiques. La métaphore utilisée plus haut, relative à la vérité issue de la croyance en Dieu et ses conséquences symboliques, trouve ici sa place en ce que le point d'origine sur lequel est censée être fondée la vérité n'est pas une garantie en soi et reste soumis à l'épreuve de sa validité.

\section{POUR LE FAUX, LE DÉBUT DE L'AMBIGUÏTÉ}

Le faux considéré sous cet aspect peut donc être abordé en opposition à la notion de vrai qui nécessite la référence à un modèle. On ne reviendra pas sur le présupposé selon lequel il n'y a pas de vrai en soi. Vraie, pourra l'être l'œuvre qui assure la continuité entre le modèle, l'auteur, la réalisation, soit une chaîne logique allant de la création à l'œuvre en passant par l'artiste.

Rappelons sans toutefois y revenir trop longuement les charnières de l'ambiguïté liées au repérage du faux. L'ambiguïté apparaît:

- lorsque le projet n'appartient pas à l'auteur mais que ce dernier en assure la réalisation;

- lorsque la réalisation s'accompagne d'un mimétisme technique qui lui garantit une assimilation avec les objets représentés; l'ensemble des pictorhèmes, les representamens, ou ce qui se regroupe sous le terme de manière, rend compte alors point par point d'un déplacement des objets, de leur transplantation sur un plan;
- lorsque l'auteur revendique l'authenticité du modèle en en contestant l'origine extérieure (c'est le cas du faussaire).

Cet aspect succinct des modalités classiques de l'ambiguité pose la question de la relation entre le faux et l'imitation. L'imitation déclarée n'est pas le faux, le faux nécessite un savoir, repérer l'imitation est un nouveau savoir.

- Il y a un modèle supposé. Quelle est la nature de ce modèle? Si sa nature est d'être la Nature, sa nature est telle qu'elle est une réplique de l'objet imité.

- La notion de mimétisme est-elle suffisante à la déclaration du faux et doit-elle être en cela différenciée catégoriquement de la notion d'imitation?

- L'imitation de la nature serait la nature en faux: d'où la difficulté qui place la nature comme valeur idéalisée au-dessus de tout jugement ou dans tous les cas comme valeur non analysable. Il s'agit de deux expressions de notre appréhension du monde. D'une part, la nature existante et, d'autre part, ses répliques multiples. Ici encore le schéma de pensée reconnu fondé sur une fonction idéale de la nature est invalidé, puisqu'on est obligé de constater qu'elle serait issue de notions telles que la constance de l'objet (en soi), l'infaillibilité à cause d'un déjà-là, une forme d'immunité épistémologique due au fait que la nature, étant ce qu'elle est sans l'intervention de l'homme, représenterait la vérité ou en serait le creuset.

S'agissant de mimétisme, cela signifie que le mode de représentation copie ou reproduit les caractères d'un objet reconnaissable, attribuable à l'expression singulière d'un auteur. On pourrait établir une comparaison diagrammatique entre modèle et mimétisme, alors qu'elle serait métaphorique entre modèle et imitation. Que peut-il surgir de ce dilemme si ce n'est la remise en cause de la notion de faux en tant que telle?

Sur le premier point, diagrammatique, le faux est à observer en tant que valeur marchande, marchandée, puisque l'implication de séduction adressée au 
spectateur, en le laissant croire aux signes symboliques du vrai, a présidé à l'exécution de l'œuvre. Nous retrouvons des éléments de réflexion comme la justification du discours sur l'objet, le renforcement d'un récit parallèle à l'objet authentique, le désir de fusion entre un modèle antérieur et son actualisation simulée. La reproduction authentique d'un tableau de Léonard de Vinci satisfera la visée esthétique de l'amateur, le croquis scientifique du squelette d'un gypaète sera éloquente pour un ornithologue, cela en tant que figures diagrammatiques de leur objet.

Sur le deuxième point, métaphorique, la notion de faux mise en cause est celle de l'authenticité de sa réplique. Joue-t-elle par rapport au modèle initial, qui a présidé à l'exécution, ou bien est-ce que cette réplique joue par rapport à une manière qui a déjà imité un modèle? Faire un faux Poussin, par exemple, est-ce imiter les mêmes paysages italiens qu'il a luimême "cadrés", sachant que cela fait partie de l'objet de création de Poussin à tous les égards, ou bien est-ce rendre compte de la plus fidèle manière de la technique de Poussin en exécutant ses moindres particularités pour créer le doute chez le spectateur? La peinture contemporaine nous offre un très bel exemple de cette approche particulière de la manière: Equipo Cronica.

Equipo Cronica est le nom donné au groupe formé dans les années soixante par deux artistes espagnols, Solbès et Valdès, qui ont fait œuvre de création ensemble. Leur goût à dénoncer l'art informel s'est caractérisé par l'emprunt de styles déjà reconnus chez d'autres peintres, et par leur report sur un même tableau. Leur position critique est très nette, mais elle le serait moins si précisément dans chacune des œuvres on ne retrouvait pas les manières de Picasso, celle de Rembrandt, de Warhol, ou de Léger, juxtaposées les unes aux autres mais organisant une image nouvelle. La totalité de la toile est constituée par les fragments imités qui créent un tout n'ayant pourtant en rien l'aspect d'un puzzle. Cela pourrait rejoindre une cohérence par imitation, si l'imitation n'était pas ici au service d'une violente critique de la peinture non figurative de l'époque. Chaque fragment est considéré comme l'icône d'un style, et chaque style est indiciaire du fragment qui lui est adjacent. Il signale à celui qui regarde que ce qui doit être vu c'est le style, mais dès lors qu'il a été perçu il est évacué au profit du tout qui organise un jeu de renvois entre les fragments. De ce point de vue, les manières qui se signalent les unes aux autres comme des indices de styles les uns par rapport aux autres ne sont plus des icônes, elles les incorporent. Sans le recours de la connaissance esthétique requise, les œuvres d'Equipo sont des icônes d'un type particulier, soit des hypoicônes ${ }^{4}$. Ce sont les qualités spécifiques de l'hypoicône qui vont être comparées lorsque la peinture soumet des objets dits semblables à l'interprétation. Si l'objet détermine le signe à l'existence, ce n'est qu'au travers du jugement perceptuel que nous pouvons le voir. Ainsi, c'est une chose bien particulière que de saisir un phénomène de ressemblance entre un objet du monde et sa représentation lorsque ces représentations sont utilisées pour ressembler à de la peinture, c'est-à-dire un objet du monde esthétique, perçu comme tel. La démarche mimétique d'Equipo n'est pas établie dans une relation diagrammatique avec des scènes du monde ordinaire qui conduisent à un effet de vérité immédiate puisqu'elle nécessite une compétence du spectateur; en revanche elle couvre une fonction symbolique qui revient à faire des peintures auxquelles l'œuvre renvoie des emblèmes de la peinture. Equipo opère là un dépassement de la notion d'imitation en désacralisant les peintures ou les peintres consacrés (puisqu'en étant imitées elles deviennent corvéables), en lui ôtant la valeur indiciaire du sujet, mais en faisant en sorte que par la compétence du spectateur les effets de manière soient interprétés symboliquement et vidés ainsi de leur contenu. Le fonctionnement de ce type d'imitation fait apparaitre une insistance de l'indice inféré par le jugement perceptuel, mais dont la validité est remise en cause par l'autocontrôle. Le spectateur compétent, en mesure d'interpréter les différentes manières qui composent la toile, opère du même coup un gommage des particularités picturales de la construction de l'unité du tableau. 
ENTRE HYPER... ET SUR...

\section{QUE DISENT LES RÉALISMES?}

En considérant ces questions, à savoir les rapports diagrammatiques ou métaphoriques que l'image-mère entretient avec l'image seconde, il est utile de préciser que deux autres mouvements de l'art contemporain illustrent clairement le cas de l'analyse iconique et replacent en cela la trichotomie de l'œuvre.

\section{L'analyse iconique}

Si l'on veut bien prendre pour sujet de l'étude le mouvement hyperréaliste (1965-1970), on constate qu'il y a un rapport diagrammatique entre les deux représentations. L'une fixant en tous points les caractères de l'autre. Tous les éléments qualitatifs de l'objet à imiter sont attribués à l'objet-peinture. Mais cet autre propose une ambiguité difficile à saisir du premier coup d'œil, car il est en plus question de l'emprunt syntaxique à un autre domaine qui est celui de la photographie. L'objet de la réalité, c'est-à-dire le modèle (celui qui est la vérité de la représentation), n'est plus mis en cause, c'est sa reproduction photographique qui est sollicitée dans l'imaginaire du spectateur. On est à un deuxième degré

d'interprétation où la duperie est fondée sur l'acquis d'une technique de représentation visuelle présupposé chez le spectateur, tel que les techniques de la peinture pourraient être sollicitées quand le sujet concerne l'imitation.

La difficulté d'analyse tient à ce que l'hyperréalisme présente une double ambiguité, d'une part dans la relation mimétique avec le modèle et, d'autre part, dans la nature qualitative des representamens qui doivent maintenir un niveau d'interprétation faussement contextualisé. Ainsi, la représentation parfaite d'une carrosserie de voiture présentée contre un mur tient le spectateur en haleine le temps du percept, mais, dans le deuxième temps des interprétants qui suit le jugement perceptuel, la confusion avec le contexte d'une rue devient impossible. Si l'objet du signe est une illusion, il est une perception interprétée par inférence logique. La chaine des interprétants successifs rétablit la vérité du contexte.
Cela mis au jour, il convient de reconsidérer le nouveau rapport d'interprétation que cela produit, car le faux de l'œuvre ne concerne pas le sujet mais les moyens de le représenter. Le mimétisme correspond à un rapport diagrammatique avec le modèle, mais ce rapport est indiciaire quant à l'habitude de représentation, puisque l'ambiguité porte sur le fait de laisser croire à la présence de l'indice.

\section{La trichotomie de l'œuvre}

On assiste à l'imitation d'un procédé technique dans l'intention de créer un subterfuge, de tromper un spectateur sur le matériau et sur l'exécution. Les mêmes cadrages, les mêmes échelles seront empruntés à l'univers de la photo, présentés de telle sorte que l'habileté picturale atteigne un paroxysme, jusqu'à ce que le visiteur s'aperçoive de l'ambiguité. La réaction ne se fait pas attendre, et il arrive fréquemment qu'il s'exclame: "Comment a-t-il fait?» C'est sans doute là que réside l'intérêt critique de la chose, à savoir un renvoi au pictural. Le comment est la question la plus précise qui puisse être posée. L'artiste utilise des gélatines photosensibles répandues sur la toile, il repeint chaque zone avec le même grain, ne négligeant aucune indication que fournit la "physiologie» de la photo. Le mot «fin» arrive quand l'ambiguité maximale est atteinte.

Dans l'univers hyperréaliste, le projet du point de vue du procès de figuration qui imite n'est pas mis en cause, ni l'auteur lui-même. Si cela se produit, la mise en cause est directement liée à l'artiste dans sa capacité à inventer un motif, car c'est de cette façon que se fera la différence esthétique, le procédé restant identique. On regroupe ainsi un Chuck Close, un Morley, ou un Hanson, tous repérés par l'originalité d'un sujet mais tous préoccupés par la restitution exacte d'un trompe-l'œil photographique. Le projet porte sur une imitation technique et non sur la mimesis d'un objet, même si celle-ci en découle. Le meilleur moyen de qualifier cette peinture est de la nommer d'abord une hypoicône. La caractérisation sémiotique du procédé est très utile puisque la distinction entre image, diagramme et métaphore 
correspond non seulement à différentes expressions picturales exprimées par les hyperréalistes, mais aussi à la distinction qualitative des pictorhèmes. On peut donner pour simple exemple la comparaison entre une œuvre de Close et une toile de Stampffli; l'un use des qualités diagrammatiques de l'icône alors que le second, en peignant des traces de pneus

surdimensionnées, use des qualités métaphoriques de l'icône ${ }^{5}$.

Le faux de l'œuvre se distingue en cela d'un faux de faussaire qui imite et le sujet et la technique. La proposition que l'hyperréalisme fait au spectateur est un faux optique qu'une approche géographique de l'œuvre effondre rapidement. Mais son sujet de première intention est l'incroyable effet de ressemblance avec une photographie. Les effets du faux concernent le support, l'ambiguité sur la réalité technique, ce qui apparaît comme une subversion au sein même de l'esthétique, puisque l'enjeu n'est pas de renvoyer à un indice dans l'atelier ou dans la salle d'exposition mais de renvoyer à un appareil absent qui contient déjà pour le peintre une image toute faite, fidèle au modèle, au point que le $19^{\mathrm{e}}$ siècle fut alarmé de la survivance des peintres après la découverte de la photographie. On se trouve en présence d'une démarche radicalement différente de celle de l'époque classique en ce que l'effet diagrammatique n'est plus porteur d'un quelconque argument de réussite sur un objet garanti par la nature et susceptible donc d'être vrai, mais d'un présupposé technique que doit connaître le spectateur pour que l'effet soit rendu perceptible. La connaissance, au moins en matière technique, intervient comme élément fondamental pour la justesse de l'interprétation. On se rend bien compte de l'écart existant entre le choix de réalisation exécuté par un peintre hyperréaliste et celui d'un peintre du $18^{\mathrm{e}}$ siècle qui reproduisait une nature morte inscrite dans l'habitude visuelle des gens. La vraisemblance était aussi dans la mise en scène. Dans le cas de l'hyperréalisme, cette vraisemblance a été aussi reproduite mais n'est ni une règle d'exécution ni un point commun aux artistes du mouvement. Le fait que nous trouvions, dans une même exposition, une scène de clochards, réalisée par un sculpteur et ressemblante à s'y méprendre, et le détail d'une voiture peint sur une grande toile avec une disproportion d'échelle, ne change rien aux qualités de compétence du spectateur. En cela, un rapprochement intéressant est à faire avec ce que Dora Vallier appelle le non-art:
Une négation se comporte en affirmation. Là où l'art est exécuté, surgit son simulacre. Les critères esthétiques réfutés sont invoqués pour justifier la réfutation. Un acte insuffisamment assumé, ni art, ni anti-art tient la scène. C'est la parodie de l'art qui se joue. Un non-art est produit, vendu et défendu par ses exégètes comme si c'était de l'art. (Vallier, 1986: 19)

Hormis le propos socio-économique, il reste que l'hyperréalisme se comporte en négation logique de la peinture (non psychologique ou esthétique, car mon propos n'est pas de prendre parti) par l'excès de son affirmation. Nous avons vu qu'il est concerné surtout par le rapport mimétique avec une technique photographique, ce qui m'a fait dire qu'il s'agissait de signe iconique, plutôt que d'une simple icône, redoublé par le fait que cette pratique imite une technique de représentation dont on demande au spectateur de la connaître parfaitement pour pouvoir la reconnaitre instantanément. Nous serons d'accord pour dire que le mimétisme décline une relation d'imitation diagrammatique (couleurs, formes, reliefs). Il en a été différemment pour le surréalisme qui, lui, avait introduit une relation d'imitation métaphorique. Mais avant d'en arriver à cette approche, regardons quelques œuvres considérées comme hyperréalistes et qui pourtant n'offrent pas d'évidentes relations de ressemblance.

\section{OBJETS SANS «CADRE» \\ L'artiste Claes Oldenburg, bien que répertorié dans le mouvement Pop Art, construit à sa manière une sorte d'hyperréalisme. Il réalise des objets de plastique gonflé qui représentent des objets ordinaires à des dimensions extraordinaires. Il faut se rappeler que le representamen ne peut que ressembler à son objet, que son objet renvoie ou non à quelque chose}


d'extérieur étant parfaitement égal. Son influence sur l'interprétation ne peut se produire que si l'esprit qui observe confond l'objet représenté avec l'objet de représentation ${ }^{6}$. Il faut être très attentif ici au fait que rien ne change du rapport diagrammatique, mais que la surdimension des objets réalisés impose de rompre avec le caractère immédiat de vérité; il ne peut y avoir d'ambiguité sur le réalisme de l'objet tout en montrant la réalité à laquelle il renvoie et qui est d'ordre esthétique. Ce sont des signes de loi de la représentation, à ce titre nommés légisignes iconiques, mais dont le jugement perceptuel ne peut rien établir d'autre que la reconnaissance d'une forme dont le déplacement de ses fonctions habituelles fait l'objet de l'hypothèse esthétique. Selon la division du signe de C.S. Peirce, la première trichotomie, celle du representamen, s'opère en trois termes, légisigne, sinsigne, qualisigne. Pour l'esthéticien et théoricien de l'art Nelson Goodman, le légisigne est aussi le «type». Nous en donnons une définition, celle de Peirce:

Un légisigne est une loi qui est un signe. Cette loi est d'ordinaire établie par les hommes. Tout signe conventionnel est un légisigne [mais non l'inverse]. Le légisigne n'est pas un objet singulier, mais un type général qui, on en a convenu, doit être signifiant. Tout légisigne signifie par son application dans un cas particulier, qu'on peut appeler sa réplique: Par exemple, le mot "the» qui apparaît d'ordinaire en anglais quinze à vingt-cinq fois par page. Il est à chaque fois le même mot, le même légisigne. Chaque cas particulier est une réplique. La réplique est un sinsigne. Ainsi, tout légisigne requiert des sinsignes. Mais ces derniers ne sont pas des signes ordinaires, comme le sont des reproductions particulières qui sont considérées comme signifiantes. Et la réplique ne serait pas signifiante sans la loi qui la rend signifiante. (C.P. 2.246, trad. Deledalle)

Peirce préférera plus tard le terme d'instance à celui de réplique. Un légisigne a besoin pour conserver son caractère de généralité d'une instanciation, et une instance de légisigne ne peut pas avoir de caractère général, puisqu'elle a une existence. On ne doit pas perdre de vue qu'il s'agit de la relation d'un signe avec sa propre nature matérielle (C.P. 8.334) ${ }^{7}$.
Quant au procédé photographique auquel on a fait allusion à propos de la peinture, il intervient comme la garantie d'une réalité dont la reproduction n'est qu'une augmentation. Dès que le spectateur prend conscience qu'il est lui-même l'objet d'une duperie, il exclut les qualités de la relation de ressemblance au bénéfice de l'indice des lieux. Lorsque Peter Klasen peint des fusibles sur toiles, à l'échelle d'une salle d'exposition et dans les couleurs qui nous sont familières, accrochées en lieu et place, un instant est nécessaire pour réfuter l'idée qu'un commissaire d'exposition ait pu laisser un tableau de fusibles installé dans une telle salle. L'imitation joue sur la familiarité avec les objets, en l'absence de cette familiarité, l'image est caduque. L'association métonymique avec la structure de l'objet rend compte du degré d'hypoiconicité de ces peintures, degré qui est le diagramme. Elles sont une illustration qualitative de la réalité représentée par des relations dyadiques analogues entre parties. C'est une relation de ressemblance entre le signe et son objet, mais le diagramme ne ressemble pas forcément à son objet; il s'agit de relations entre parties. Le diagramme est une des divisions de la relation monadique qui se rapportent aux trois distinctions phénoménologiques (image, diagramme, métaphore):

On peut en gros diviser les hypoicônes suivant le mode de priméité auquel elles participent. Celles qui font partie des simples qualités ou premières priméités, sont des images; celles qui représentent les relations, principalement dyadiques ou considérées comme telles, des parties d'une chose par des relations analogues dans leurs propres parties, sont des diagrammes; celles qui représentent le caractère représentatif d'un representamen en représentant un parallélisme dans quelque chose d'autre, sont des métaphores.

(C.P. 2.277, trad. Deledalle)

\section{UN AUTRE CAS, CELUI DU SURRÉALISME}

L'élargissement à la métaphore dans la trichotomie de l'icône est d'un intérêt particulier pour les œuvres du mouvement surréaliste. Les tableaux surréalistes entretiennent avec leurs objets des relations de ressemblance. La question qu'ils soulèvent est celle de 
la mise en contexte de ces objets. De fait, les objets en eux-mêmes peuvent être des signes iconiques comme le sont les peintures hyperréalistes, mais la proximité d'autres objets qui apparemment n'ont rien à faire ensemble fait éclater le rapport de ressemblance. Ainsi, pris isolément, chaque signe iconique est perçu au niveau mimétique (comme on peut le rencontrer chez Magritte) mais, dès lors que le contexte est pris en considération, la relation d'objet à objet est métaphorique. Elle l'est quant à l'impossibilité réelle d'une telle proximité (un parapluie et une machine à coudre), mais elle l'est aussi quant à la déformation occasionnelle de certains de ces objets (les montres molles de Dali). Les caractères de discrimination demeurent présents dans la mesure où l'on reconnaît qu'il s'agit d'une montre, mais rien ne peut autoriser à en imaginer le caractère de vérité puisqu'elle est molle, et installée sur des branches d'arbre. Cette question est importante en ce qu'elle rejoint la question du réalisme en peinture. On peut, en effet, rapprocher cela de certaines peintures de Parmigiano ou de Renoir. Les déformations imposées par l'invenzione dont faisait preuve Parmigiano (allongement du cou des personnages, figure serpentine, etc.) ou le spécifiquement pictural (corps qui n'a pas l'air de s'appuyer sur le sol mais semble flotter) rejoignent cette impossibilité de réalité. Les femmes de Renoir ont des cuisses qui ne peuvent s'articuler à leur corps, les madones de Parmigiano ont plus l'élégance de poupées étirées et surnaturelles, comme le seraient des femmes girafes sans collier, que l'allure des femmes de la réalité.

Ces incohérences physiques signifient précisément que la question posée par la peinture n'est pas celle de la réalité mais celle du réel. Pour le sujet qui nous préoccupe, il est intéressant de montrer que le peintre réalise une mise en scène; il fabrique un réel qui est le sien, mais les objets qu'il représente étant déjà inscrits dans un rapport de ressemblance avec la réalité, il induit donc chez le spectateur une interprétation qui introduit le faux; un faux de l'objet sur le but à atteindre, faux qui caractérise précisément le projet. Or, les signes iconiques proposés dans les toiles de Magritte ou de Dali entretiennent une relation de ressemblance minimale, c'est-à-dire une quantification d'indices réduits à ce qui illustre leur structure. Relation pour le moins instable puisqu'on ne peut y retrouver ni logique fonctionnelle (une femme oui, mais à tiroirs), ni positionnement dans l'espace liés à une quelconque réalité (une pomme oui, mais occupant tout le volume d'une pièce).

Il en est de même lorsqu'on observe chez Molière le discours des Précieuses ridicules, dans lequel est particulièrement évoqué le mot «fauteuil", remplacé par les "commodités de la conversation». La toile de Dali, qui montre une femme dont le corps est fabriqué comme celui d'une commode, accuse un trajet tout aussi détourné pour suggérer que la femme est à tiroirs. Cela tient au fait que le surréalisme présente une métaphore complexe. Métaphore présente comme celle d'un imaginaire traduit dans le tableau mais qui n'est pas directement opérationnelle. Voulant exprimer la multiplicité des humeurs féminines, Dali, on peut le supposer, l'a traduite par une suite de tiroirs mais, en l'appelant Vénus, il déstabilise les canons de beauté rattachés à l'idée de femme: sa Vénus est plutôt tortueuse et languissante et les tiroirs sont de véritables blessures. La limite de la métaphore est là. Il faut donc être très prudent et connaître l'univers du peintre pour conclure à «ce qu'il aurait voulu dire». D'où la nécessité de comprendre ces objets dans la relation métaphorique qu'ils entretiennent entre eux jusqu'à la limite des interprétants possibles. L'équipement culturel de l'interprète peut prétendre à rapprocher cette Vénus de certaine peinture de la Renaissance italienne ( $L a$ Madone au long cou de Parmigiano), utilisant donc les artifices spécifiques de l'époque à des fins subversives en allant jusqu'à instrumentaliser le corps de la femme.

C'est parce que ce déplacement est bel et bien efficace que la réaction du spectateur peut être immédiate et que l'on doit donc ici encore conclure à la métaphore (accusée de surcroît par le titre). Dans un article consacré à Magritte, j'avais proposé de nommer cela une "auto-métaphore» de l'image, dans la mesure où celle-ci contient son propre déplacement 
par le traçage d'objets éloignés symboliquement, ou ne fonctionnant pas ensemble, dans un univers non analogue, sur une même surface et à la «lecture» de tous. Tout se passe comme si chaque objet s'hypostasiait dans un autre; on voit alors la limite laissée à l'imaginaire du spectateur: Magritte est conduit à désirer substituer le sien propre à celui du spectateur $^{8}$.

\section{CONCLUSION}

Duchamp propose l'objet à la place de sa représentation en obtenant pour effet un art rétinien remplacé par la sollicitation de l'intelligence. Picasso avait fait cela en introduisant le collage dans ses peintures. C'est le même Duchamp qui affirme: «en dehors de l'histoire, rien ne permet d'affirmer comme une vérité: ceci est une œuvre d'art». Et pour cause. Dès lors que la compétence à reproduire, l'excellence à imiter ne sont plus répertoriées comme critères de qualité esthétique, la sacralisation du savoir-faire disparaît. L'hyperréalisme et le surréalisme sont deux types de production artistique qui, tout en exhibant une qualité technique performante, contredisent cette capacité et renvoient le spectateur au réel, c'est-à-dire au pictural.

En d'autres termes et pour caractériser les deux mouvements que j'ai fait intervenir dans mon analyse, l'apparence de fausseté de l'hyperréalisme est à mettre en relation avec la réalisation technique; l'objet $\mathrm{du}$ signe rend compte d'une ambiguité iconique du mode de réalisation. La représentation d'objets est mise en scène de manière mimétique par rapport à l'original. La connaissance de l'objet par le spectateur est sollicitée au premier chef pour donner le change à l'ambiguité. Il est la réplique d'un objet existant et, en tant que classe de signes, un légisigne iconique rhématique de l'original ${ }^{9}$.

Pour le surréalisme, l'objet du signe détermine la forme d'un déplacement contextuel. Le faux du surréalisme est à mettre en relation avec le projet. Les objets représentés entretiennent une ressemblance avec l'original, mais il s'agit de simples traits discriminants qui ne précisent pas l'objet d'origine. Si les objets entretiennent une ambiguïté avec leur original, celle-ci existe quant à leur fonction; ces objets sont reconnaissables mais inutilisables ou inopérants. Leur mise en contexte n'a aucun caractère de véridicité. Le faux du faussaire, celui qui nous est familier en tant que faux, s'adresse plutôt à l'auteur et est à mettre en relation avec lui. L'intention de duperie se manifeste par le désir d'adresser ce projet singulier au spectateur. C'est une œuvre qui est mise pour quelque chose d'autre. Elle est un representamen de l'original ou, plus précisément, un légisigne indiciaire rhématique. En tout état de cause, il n'y a pas d'en-soi, même dans l'imitation.

Si l'on veut bien se rappeler ce qui a été formulé à propos de l'hyperréalisme, et du commentaire rapporté par Dora Vallier, je suis tentée de conclure que toute peinture est une fiction, une fiction qui édifie le réel. La peinture ne peut être qu'un faux de la réalité, mais une fiction que le spectateur prendra pour vraie. Ce processus d'insistance dans la déclaration de l'impossible authentique me semble être l'expression du faux d'un faux, identique à l'exclamation «nom de nom!».

La conclusion qu'on pourrait en tirer est que le faux comme "clause» esthétique n'existe pas en dehors du sujet. S'il existe, c'est dans la négation de l'invention, c'est-à-dire d'un dispositif créatif qui gouverne la construction de l'œuvre et qui, dans le cas de la mimesis, est emprunté à quelqu'un d'autre.

Dans notre analyse, le faux concerne la copie diagrammatique d'un motif impliqué en tant que modèle, c'est-à-dire en tant que sujet. En ce sens, l'hyperréalisme - autant que le surréalisme - n'est pas subordonné à l'imitation du modèle pour le substituer à la réalité tout en convainquant de la performance technique du peintre, mais il utilise et dépasse la saisie de la ressemblance avec lui-même pour renvoyer spécifiquement au pictural. Pour le surréalisme, il suffit que l'objet soit reconnu. Pour l'hyperréalisme, l'imitation est un procès de seconde intention dans la mesure où elle ne fait que servir une cause qui lui est quasiment étrangère et psychologique: le moment fugace du mensonge et l'ambiguité auxquels le 
spectateur se fait prendre un instant. La peinture n'a plus alors la même stabilité, elle n'est pas donnée au regard pour qu'on en admire tous les caractères, elle propose un saisissement fugace de l'émotion. Après quoi le regard est inductivement analytique et l'œuvre perd sa fonction provocatrice. En tant qu'objet d'une exécution singulière, le faux n'existe pas.

J'annonçais que l'étude de cette question commençait par celle des composantes iconiques. Létude sémiotique a montré que la perception des icônes s'effectue dans une relation immédiate à l'univers des qualités par le jugement perceptuel et que les icônes nouvelles, formées dans l'esprit du spectateur, sont préscindées ou précissées d'une connaissance collatérale que le spectateur a des objets représentés ${ }^{10}$.

La sensibilité ne peut faire loi dans la mesure où elle ne représente qu'un jugement individué, mais elle reste indispensable à la constitution du jugement comme principe immédiat de discrimination, lorsqu'elle a été travaillée par la connaissance.

\section{NOTES}

1. Le terme de pictorhème a été formé sur le radical pict-(image) et la désinence sémiotique rhème (qui a priori ne dit rien). Un pictorhème est donc un élément constitutif de l'œuvre sans autre considération interprétative.

2. "Une icône est un signe qui posséderait le caractère qui le rend signifiant, même si son objet n'existait pas. Exemple: un trait au crayon représentant une ligne géométrique. Un indice est un signe qui perdrait immédiatement le caractère qui en fait un signe si son objet était supprimé, mais ne perdrait pas ce caractère s'il n'y avait pas d'interprétant. Exemple: un moulage avec un trou de balle dedans comme signe de coup de feu; car sans le coup de feu il n'y aurait pas eu de trou; mais il y a un trou là, que quelqu'un ait l'idée de l'attribuer à un coup de feu ou non. Un symbole est un signe qui perdrait le caractère qui en fait un signe s'il n'y avait pas d'interprétant. Exemple: tout discours qui signifie par le seul fait que l'on comprenne qu'il a cette signification ». (2.304, trad. Deledalle).

3. «Un rhème est un signe qui, pour son interprétant, est un signe de possibilité qualitative" (2.250). "Un signe dicent est un signe qui, pour son interprétant, est un signe d'existence réelle " (2.251). Un argument est un signe qui, pour son interprétant, est un signe de loi (2.252); trad. Deledalle.

4. «Seule une possibilité est une icône, purement et en vertu de sa qualité; et son objet ne peut être qu'une priméité. Mais un signe peut être iconique, c'est-à-dire peut représenter son objet principalement par sa similarité, quel que soit son mode d'être. S'il faut un substantif, un representamen iconique peut être appelé une hypoicône. Toute image matérielle, comme un tableau, est largement conventionnelle dans son mode de représentation; mais en soi, sans légende ni étiquette, on peut l'appeler une hypoicône». (2.276, trad. Deledalle).

5. Image, diagramme et métaphore font partie de la trichotomie de l'icône ou plus précisément de l'hypoicône, dont la définition est donnée plus loin dans le texte.

6. On peut retrouver les précisions sémiotiques dans Caruana, 1991, p. 104 sq.

7. Si le légisigne est de type général, sa réplique, la forme utilisée par Viallat l'est toujours dans un cas particulier. En tant que réplique, elle est un sinsigne, mais ce qui la rend signifiante est la loi. Cette loi détermine l'interprétation du symbole par référence à cet objet. (cf. 2.249). Le symbole est donc lui-même un type général ou une loi, c'està-dire un légisigne. À ce titre, il agit par l'intermédiaire d'une réplique: ainsi la réalité des autres formes consiste en ce qu'elles se conforment à elle, c'est-à-dire à la réplique, en tant que matérialisation du symbole. Cf. Caruana, 1989: 772.

8. J'entends par là que les images élaborées par Magritte engendrent elles-mêmes leur propre métaphore et orientent l'imaginaire précisément vers des solutions contenues dans le déplacement proposé. L'ouverture habituellement induite par la métaphore est ici enkystée dans le tableau même sous la gouverne de ces images hypostasiées. Il sera plus aisé de différencier ces deux expressions d'une métaphore en prenant pour exemple, d'un côté une métaphore ordinaire telle que "la mer ondule", équivalant à "une plaine d'eau balancée par le vent ", et de l'autre celle d'une découpe en forme d'oiseau dont la matière est le ciel, qui serait donc intitulée "oiseau de ciel», sans aucune autre possibilité de glissement métaphorique. Une première conclusion fournie par l'analyse rappelle que ce que j'ai décrit comme une auto-métaphore correspond à un guidage du regard dans un univers où le peintre ne souhaite pas de débordements imaginaires du spectateur, débordements qui l'amèneraient à être hors sujet. Par ailleurs, c'est le sujet en tant que mise en scène qui représente les conditions de possibilité de l'interprétation. La mise en scène représente ces conditions de possibilités pour la sémiose dégénérée. Cf. Caruana, 1996.

9. Il s'agit de la dimension pragmatique du signe décrite par Peirce. Voir l'ouvrage de G. Deledalle, 1979: 79sq.

10. On doit rapprocher de la présupposition ce mode de séparation de la pensée analysé par Peirce (1992: 340-342).

\section{RÉFÉRENCES BIBLIOGRAPHIQUES}

CARUANA, F. [1996]: "Imaginagritte», Degrés, 88, a1-a21;

[1991]: Problèmes d'interprétation en peinture, Thèse de doctorat, Université de Perpignan, Lille;

[1989]: «La stratégie du clone", Revue S, vol. I à IV, 763-777.

DELEDALLE, G. [1979]: Théorie et pratique du signe, introduction à la sémiotique de Charles S. Peirce, Paris, Payot.

PEIRCE, C. S. [1992] : À la recherche d'une méthode, (sous la dir. de M.

Balat et J. Deledalle-Rhodes), Perpignan, P.U.P.;

[1978] : Écrits sur le signe, rassemblés, traduits et commentés par G. Deledalle, Paris, Seuil.

VAllieR, D. [1986]: Art, anti-art, non-art, Caen, Éd. de l'échoppe.

\section{OEUVRES CITÉES}

TORCZYNER, H. [1978]: Magritte, le véritable art de peindre, Paris, Draeger. Les Hyperréalistes américains, Paris, Éd. Filipacchi, 1973.

Salvador Dali, rétrospective 1920-1980, Catalogue d'exposition du Musée National d'art moderne du Centre Georges Pompidou, Paris, 1980. Pop art, Paris, Éd. Fernand Hazan, 1964 (pour Claes Oldenburg). 